\title{
Efecto del uso de dentifricos aclaradores sobre la estructura y superficie del esmalte dental.
}

\author{
Jairo Mariel Cárdenas y Francisco Javier Gutiérrez Cantú \\ Maestría en Ciencias Odontológicas, Facultad de Estomatología, Universidad Autónoma \\ de San Luis Potosí, México.
}

Palabras clave: esmalte; dentífrico; aclaramiento dental.

Resumen. A causa de la insatisfacción que existe por parte de algunos pacientes en relación con el color o tono de sus órganos dentarios, casas comerciales han diseñado y producido formulaciones de dentífricos con la intención de obtener un efecto de aclaramiento por parte de estos. El objetivo de este estudio fue evaluar el efecto de dentífricos aclaradores sobre la dureza, rugosidad y superficie del tejido adamantino. Se recolectaron y analizaron 48 incisivos permanentes sanos extraídos por enfermedad periodontal, se dividieron en seis grupos de estudio: Grupo 1: Sin cepillado, Grupo 2: Control (Sin pasta dental) y 4 experimentales (Colgate Triple Acción, Colgate Luminous White, Crest y Crest 3D). Se analizó la estructura adamantina con un micro durómetro, la rugosidad con microscopia de fuerza atómica y la superficie con microscopia electrónica de barrido. Para el análisis estadístico se utilizó el software MINI$\mathrm{TAB}$ versión 19. Se identificaron diferencias significativas en el aumento en la microdureza dental, cambios topográficos en la superficie adamantina y diferentes patrones de pulido producidos por el uso de las diferentes pastas dentales empleadas. 


\title{
Effect of the use of lightening toothpastes on the structure and surface of the dental enamel.
}

\author{
Invest Clin 2021; 62 (1): 63-72
}

Key words: enamel; dentifrice; dental bleaching.

\begin{abstract}
Because of the dissatisfaction that exists on the part of some patients in relation to the color or tone of their dental organs, commercial companies have designed and produced toothpaste formulations with the intention of obtaining a whiter teeth effect. The objective of this study was to evaluate the effect of whitening toothpastes on the hardness, roughness and surface of the adamantine tissue. Forty-eight healthy permanent incisors, extracted due to periodontal disease, were collected and analyzed and they were divided into six study groups: Group 1: No Brushing, Group 2: Control (No Toothpaste) and four Experimental (Colgate Triple Action, Colgate Luminous White, Crest and Crest 3D). Adamantine structure was analyzed with a micro hardness tester, roughness with atomic force microscopy and the surface with scanning electron microscopy. For the statistical analysis we used the software MINITAB version 19. Significant differences were identified in the increase in dental micro hardness, topographic changes in the adamantine surface and different polishing patterns produced by the use of the different toothpastes used.
\end{abstract}

Recibido: 22-07-2020 Aceptado: 18-12-2020

\section{INTRODUCGION}

La mayoría de los productos de blanqueamiento dental funcionan de una o dos maneras, ya sea mediante el aclarado de los dientes o mediante la eliminación y el control de la mancha dental extrínseca o su color intrínseco (1-2). Los mecanismos que producen un cambio en la percepción del color del diente se subdividen en tres fases: primero, el movimiento del agente blanqueador en la estructura del diente; segundo, la interacción del aǵente blanqueador con las moléculas colorantes; y tercero, la alteración de la superficie de la estructura del diente de modo que refleje la luz (3). A causa de la insatisfacción que existe por parte de algunos pacientes en relación con el color o tono de sus órganos dentarios (OD), diversas casas comerciales han diseñado y producido formulaciones de dentífricos con la intención de obtener un efecto de aclaramiento por parte de estos. Algunos de los ingredientes funcionales clave son los componentes abrasivos, los cuales se han diseñado para obtener un efecto de remoción de los pigmentos extrínsecos y prevenir la reincidencia de estos (4-5). Por lo tanto, no solo el ingrediente activo, sino también los agentes abrasivos de una pasta dental son importantes. Las investigaciones sobre la eficacia de las cremas dentales revelan una amplia gama de efectos y diferentes grados de protección. También es posible que la fracción particulada en una formulación de pasta de dientes sea un importante modulador de la eficacia. Tales partículas son en su mayoría sílice hidratada (6-7). Existe una gran variedad de productos 
que se emplean en el hogar sin la necesidad de la asistencia de un profesional de la salud; es posible que a causa de un empleo excesivo o inadecuado de estos se produzca daño o alteraciones sobre las estructuras orales, incluyendo la superficie del esmalte. Alguno de los compuestos usualmente empleados y que son parte de la formulación en los dentífricos incluyen carbonato cálcico, fosfato dicálcico dihidratado, sílica hidratada, bicarbonato de sodio, alúmina y perlita (8). Las pastas dentales se pueden formular con diferentes sistemas abrasivos, dependiendo de su aplicación clínica prevista. Esta formulación puede afectar su efectividad y seguridad $\mathrm{y}$, por lo tanto, requiere una comprensión adecuada (9). Los abrasivos son componentes esenciales de la formulación del dentífrico, ya que la acción física es el principal mecanismo responsable de la limpieza dental y son responsables del grado de desgaste en el tejido adamantino (10-11), aunque también es el mecanismo de desgaste abrasivo más relevante que afecta a la zona cervical, con una relación claramente establecida (12). Existen estudios que refieren que los productos aclarantes pueden producir quemaduras, sensibilidad transitoria o permanente, cambios en la microestructura dental como reducción de microdureza e incremento de la rugosidad de la superficie de los OD (13-14).

En la actualidad ha surgido una gran polémica en relación con los productos que remueven las manchas y blanquean la superficie dental. Se han identificado dentífricos que usan métodos físicos (abrasivos) o químicos (agentes de superficie y oxidantes) y son de uso común entre particulares y profesionales del área de la salud. Su presentación puede comercializarse en gel o pasta dental con un método de 2 o 3 pasos en los cuales se aplican contenidos de peróxido de hidrógeno o carbamida como el ingrediente blanqueador o desmanchador. En el desgaste dental es importante mencionar que la presencia de agentes abrasivos en los dentífricos blanqueadores y el uso sin con- trol y supervisión de un odontóloǵo pueden generar alteraciones en tejidos dentales, así como recesión gingival, abrasión cervical e incluso hipersensibilidad en la dentina.

Los dentífricos comerciales más empleados en la industria mexicana son Colgate y Crest, ambos se caracterizan por tener diversas presentaciones con propiedades aclarantes. El objetivo de este estudio fue evaluar el efecto de dentífricos aclaradores sobre: el grado de resistencia que posee un mineral al ser rayado o penetrado por otro material (dureza), el conjunto de irregularidades de la superficie dental (rugosidad) y el aspecto exterior de la parte más externa del tejido adamantino (superficie).

\section{MATERIALES Y MÉTODOS}

El estudio se realizó en el laboratorio de nanomateriales de la Maestría en Ciencias Odontológicas de la Facultad de Estomatología de la Universidad Autónoma de San Luis Potosí, México. Se analizaron 48 incisivos permanentes sanos extraídos por enfermedad periodontal; las muestras fueron almacenadas en agua destilada a $-4^{\circ} \mathrm{C}$. Se dividieron en seis grupos de estudio: Grupo 1: Sin cepillado, Grupo 2: Control (sin pasta dental) y 4 experimentales (Colgate Triple Acción, Colgate Luminous White, Crest y Crest 3D. Se utilizó eepillo dental Colgate ${ }^{\circledR}$ Ultra Soft con cerdas medianas para todos los grupos de estudio, con la técnica de cepillado Bass modificada. Los OD estuvieron soportados en una base de acrílicos sujetos a una prensa metálica. Se realizaron cepillados dentales de forma manual por la misma persona para cada uno de los grupos en la cara labial desde la porción cervical hasta la incisal con diferentes números de barridos simulando el tiempo de exposición del dentífrico aclarante al tejido adamantino que se usa de manera cotidiana ( 3 veces al día cinco cepilladas por segmento) los cuales incluyen: 0/0, 7/105, 14/210, 28/420 (días/ cepillada, respectivamente). La estructura

Vol. 62(1): 63 - 72, 2021 
adamantina se analizó con los siguientes equipos: para el análisis de la dureza del esmalte dental, un microdurómetro (SHIMA$\mathrm{DZU}^{\circledR}$ ); la rugosidad, con microscopia de fuerza atómica $\left(\right.$ NANOSURF $\left.^{\circledR}\right)$ (Fiǵ. 1) y la superficie, con microscopia electrónica de barrido (JSM-6510 ${ }^{\circledR}$ ) (Fiǵ. 2). Para el análisis estadístico se utilizó el software MINITAB versión 19, se determinó la estadística descriptiva y se analizó la normalidad de las variables con la prueba de Shapiro Wilk. Al ser un estudio no paramétrico se usó la prueba de U Mann Whitney comparando las medianas para determinar la significancia estadística con intervalos de confianza al 95\%.

\section{RESULTADOS}

Se determinó la mediana, valores mínimos y máximos para la rugosidad y microdureza en los diferentes tiempos de análisis para cada grupo de estudio (Tabla I).

Cuando se analizó la rugosidad se encontró diferencia significativa al realizar cepillado dental con respecto a no realizarlo, observándose pulido de la superficie adamantina producida por el cepillado dental en todos los tiempos de estudio. A los 7 días, comparado con el Grupo 2 (Control Sin pasta dental), se observó una menor pérdida de la rugosidad con el uso de crema dental, particularmen-

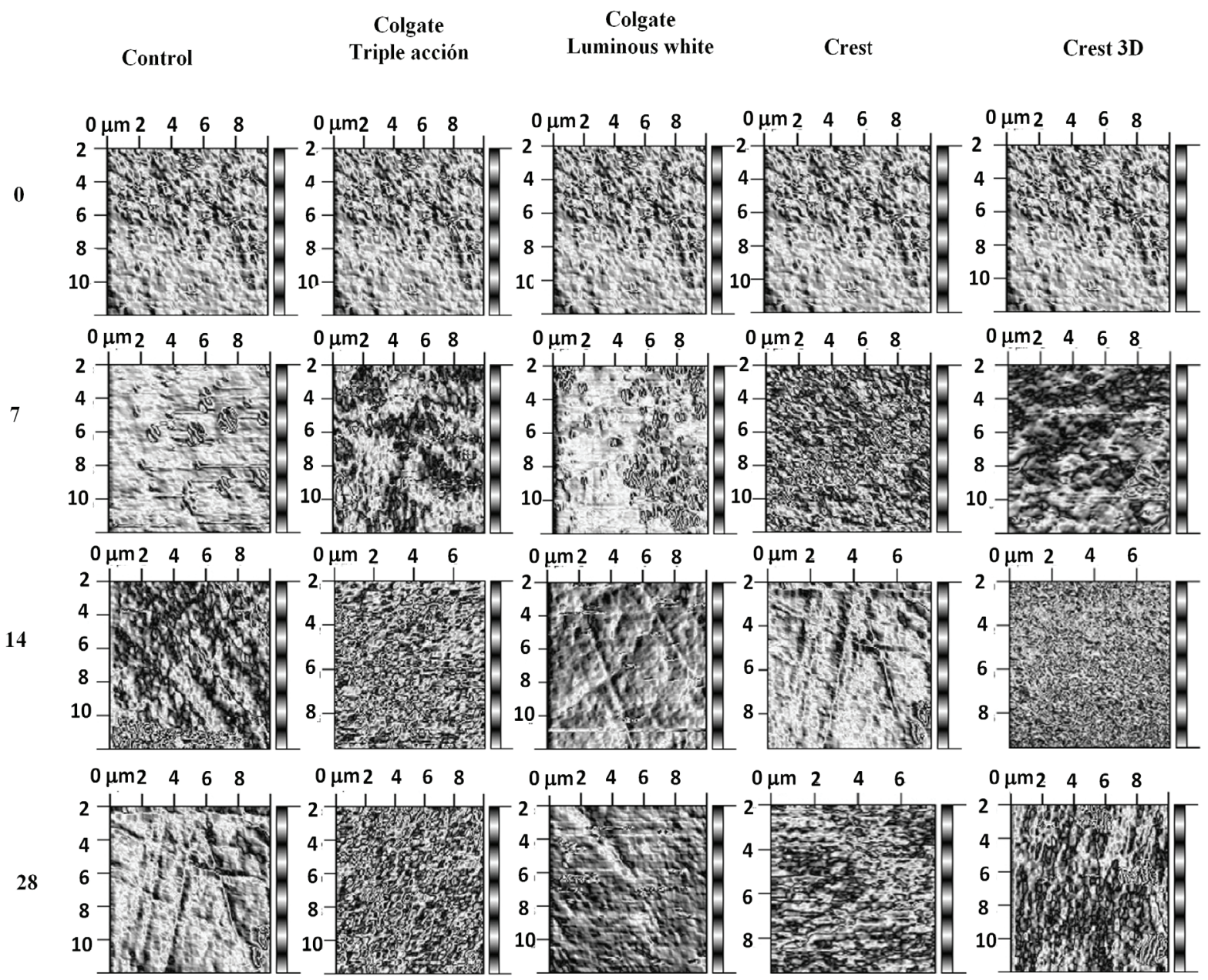

Fig. 1. Microscopia de fuerza atómica. 


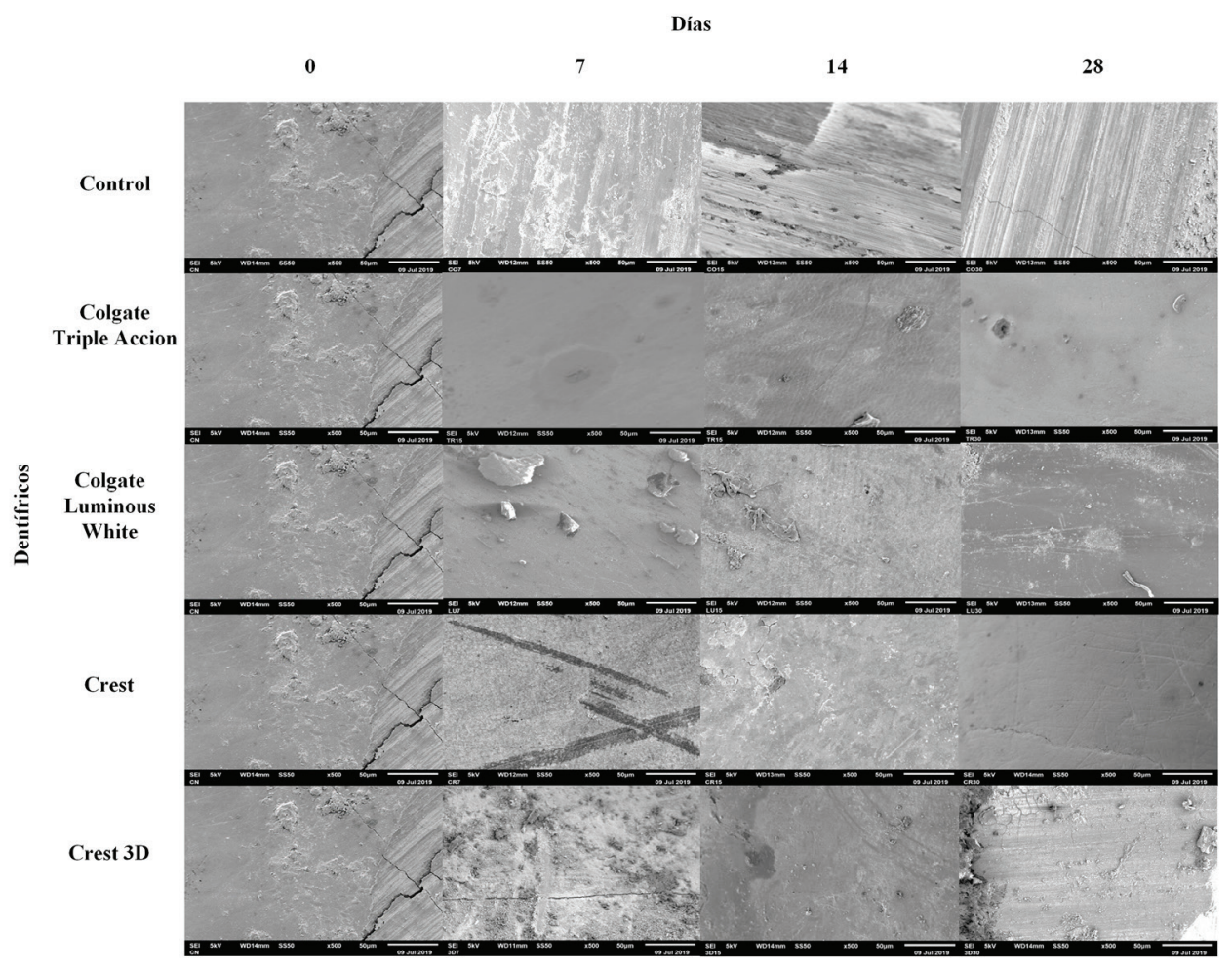

Fig. 2. Microscopia electrónica de barrido.

TABLA I

ESTADÍSTICA DESCRIPTIVA DE LA RUGOSIDAD Y MICRODUREZA EN LOS DIFERENTES TIEMPOS DE ANÁLISIS PARA CADA GRUPO DE ESTUDIO.

\begin{tabular}{|c|c|c|c|c|c|c|c|}
\hline \multirow[b]{2}{*}{ Tiempo (días) } & \multirow[b]{2}{*}{ Pasta } & \multicolumn{3}{|c|}{ Rugosidad } & \multicolumn{3}{|c|}{ Microdureza } \\
\hline & & Mediana & Mínimo & Máximo & Mediana & Mínimo & Máximo \\
\hline 0 & Sin cepillado & 313,2 & 174,5 & 741,60 & 237,0 & 75,4 & 367 \\
\hline \multirow[t]{5}{*}{7} & Control & 17,91 & 6,950 & 34,910 & 294,0 & 180 & 362 \\
\hline & Colgate Triple Acción & 14,79 & 4,710 & 55,070 & 279,0 & 221 & 442 \\
\hline & Colgate Luminous White & 21,96 & 6,020 & 121,62 & 305,5 & 173 & 423 \\
\hline & Crest & 31,73 & 14,44 & 79,150 & 310,0 & 159 & 397 \\
\hline & Crest 3D & 29,02 & 18,11 & 106,28 & 274,0 & 222 & 412 \\
\hline \multirow[t]{5}{*}{14} & Control & 20,84 & 6,400 & 44,530 & 320,0 & 116 & 378 \\
\hline & Colgate Triple Acción & 56,40 & 12,30 & 250,70 & 275,0 & 78,7 & 451 \\
\hline & Colgate Luminous White & 22,18 & 10,02 & 57,880 & 315,5 & 202 & 402 \\
\hline & Crest & 24,57 & 6,200 & 96,470 & 309,0 & 221 & 467 \\
\hline & Crest 3D & 20,60 & 10,80 & 133,10 & 264,0 & 149 & 423 \\
\hline \multirow[t]{5}{*}{28} & Control & 24,30 & 14,20 & 95,020 & 190,0 & 57,6 & 290 \\
\hline & Colgate Triple Acción & 43,30 & 14,25 & 174,09 & 317,5 & 229 & 373 \\
\hline & Colgate Luminous White & 63,24 & 19,06 & 111,72 & 251,5 & 103 & 338 \\
\hline & Crest & 41,67 & 27,61 & 78,680 & 290,5 & 158 & 365 \\
\hline & Crest 3D & 40,80 & 13,21 & 80,300 & 324,0 & 96,8 & 532 \\
\hline
\end{tabular}

Vol. 62(1): $63-72,2021$ 
te con las marcas Crest y mayor abrasión por la pasta Colgate Triple Acción. A los 14 días se identificó menor abrasión de la superficie adamantina con el uso de Colgate Triple Acción, mientras que a los 28 días tal efecto se observó con Luminous White, encontrándose un mayor pulido dental con el grupo Control (cepillado sin pasta). Con respecto a la microdureza no se identificó diferencia a los 7 días entre usar o no pastal dental. A los 14 días al comparar con el grupo de sin cepillado se observaron diferencias con todas las pastas dentales no así con Colgate Triple Acción, mientras que a los 28 días no se identificaron diferencias con Luminous White, y se observó un aumento de la microdureza con las pastas dentales Crest 3D y Colgate Triple Acción (Figss. 1 y 2, Tabla II).

Se realizó la comparación de la rugosidad y microdureza entre los tiempos de exposición para cada grupo de estudio. En el grupo control (Sin pasta dental) se observaron diferencias en la rugosidad para todos los tiempos de estudio, no así la microdureza. Así, en este grupo se observó la mayor pérdida de la rugosidad durante la primera semana de cepillado, mientras que la microdureza disminuyó a partir de los 14 días. Con respecto a los grupos experimentales, la pasta dental Colgate Triple Acción inicialmente mostró una disminución en la rugosidad a los 7 días, seguido de un patrón irregular en la superficie dental a los 14 días, no así a los 28 días cuando nuevamente se observó un pulido dental, mientras que los mayores cambios en la microdureza se identificaron en la primera semana. La pasta Luminous White mostró el mayor pulido dental durante los primeros 7 días, pero en las siguientes semanas se produce una mayor rugosidad, mientras que la microdureza disminuyó a partir de los 14 días. Con respecto a la rugosidad de la pasta Crest,

TABLA II

COMPARACIONES DE LA RUGOSIDAD Y MICRODUREZA ENTRE LOS GRUPOS DE ESTUDIO EN DIFERENTES TIEMPOS.

\begin{tabular}{ccccccc}
\hline & \multicolumn{2}{c}{7 días } & \multicolumn{2}{c}{14 días } & \multicolumn{2}{c}{28 días } \\
\cline { 2 - 7 } Grupos de estudio & Rugosidad & Microdureza & Rugosidad & Microdureza & Rugosidad & Microdureza \\
\hline Se vs Co & 0,0001 & 0,1173 & 0,0001 & 0,0025 & 0,0001 & 0,0001 \\
Se vs Tr & 0,0001 & 0,0498 & 0,0001 & 0,8494 & 0,0001 & 0,0018 \\
Se vs Lu & 0,0001 & 0,0354 & 0,0001 & 0,0136 & 0,0001 & 0,8868 \\
Sc vs Cr & 0,0001 & 0,0354 & 0,0001 & 0,0079 & 0,0001 & 0,0368 \\
Se vs 3D & 0,0001 & 0,0340 & 0,0001 & 0,0040 & 0,0001 & 0,0004 \\
Co vs Tr & 0,6464 & 0,8248 & 0,0009 & 0,1208 & 0,0642 & 0,0001 \\
Co vs Lu & 0,1249 & 0,4196 & 0,7160 & 1,0000 & 0,0020 & 0,0001 \\
Co vs Cr & 0,0011 & 0,3189 & 0,4107 & 0,2543 & 0,0082 & 0,0001 \\
Co vs 3D & 0,0005 & 0,5063 & 0,6127 & 0,1890 & 0,0279 & 0,0001 \\
Tr vs Lu & 0,0290 & 0,6924 & 0,0017 & 0,0619 & 0,1249 & 0,0124 \\
Tr vs Cr & 0,0016 & 0,5689 & 0,0082 & 0,4017 & 0,7042 & 0,0846 \\
Tr vs 3D & 0,0006 & 0,7396 & 0,0462 & 0,2353 & 0,8868 & 0,1454 \\
Lu vs Cr & 0,5583 & 0,9369 & 0,6925 & 0,9369 & 0,0500 & 0,2354 \\
Lu vs 3D & 0,2114 & 0,8494 & 0,7517 & 0,2056 & 0,0689 & 0,0049 \\
Cr vs 3D & 0,4765 & 0,9118 & 0,8619 & 0,4383 & 0,7397 & 0,0038 \\
\hline
\end{tabular}

$\mathrm{R}=$ Rugosidad, $\mathrm{M}=$ microdureza, $\mathrm{Se}=$ Sin cepillado, $\mathrm{Co}=$ Control, $\mathrm{Tr}=$ Colgate Triple Acción, Lu= Colgate Luminous White, $\mathrm{Cr}=$ Crest, $\mathrm{y} 3 \mathrm{D}=$ Crest 3D. 
se observó su disminución durante los primeros 14 días creando nueva rugosidad a los 28 días; la mayor microdureza se identificó en los primeros 7 días, manteniendo sus valores en las siguientes semanas. La rugosidad de la pasta Crest 3D mostró un patrón similar al de la pasta Crest, mientras que la mayor microdureza se identificó de los 14 a los 28 días (Figs. 1 y 2, Tabla III).

\section{DISCUSIÓN}

En el presente trabajo se evaluó la superficie del esmalte dental, expuesta a dentífricos aclaradores en diferentes tiempos. Se identificaron cambios debido al arrastre mecánico del cepillado dental en conjuntamente con los abrasivos que contienen las pastas dentales aclaradoras, las cuales generan modificaciones en la estructura y la morfohistología de los OD.

Maden y cols. en 2018 realizaron una evaluación in vitro del efecto de las pastas dentales con fluoruro, xilitol, probióticos y blanqueadores sobre la rugosidad y la microdureza del esmalte en dientes permanentes. Se observó diferencia estadísticamente significativa entre los valores de microdureza antes y después del cepillado en todos los grupos, excepto el grupo cepillado con pasta dental Colgate Maxfresh. Se encontró una reducción significativa en la microdureza en muestras de esmalte cepilladas con las pastas de dientes. La diferencia en la reducción de microdureza en las muestras de esmalte de estas pastas dentales no fue estadísticamente significativa (15). En este estudio se utilizaron pastas blanqueadoras diferentes al estudio de Maden y col., pero con ingredientes similares. En la presente investigación se realizó el cepillado manual con cepillo convencional y cerdas medianas a diferencia de ellos que lo efectuaron con cepillo eléctrico con cerdas suaves, así como también realizaron un pulido previo al esmalte dental, y su tiempo de cepillado fue menor al de nosotros (una semana), lo anterior pudo influir en los cambios de la estructura del esmalte dental posterior al uso prolongado de las diversas técnicas empleadas tal vez debido a las diferencias de los movimientos propios entre el cepillado eléctrico y el convencional; El uso de cerdas medianas culturalmente aún persiste de manera cotidiana en la población y a diferencia de las cerdas suaves probablemente pueda producir un desgaste con patrones distintos y mayor fuerza a la estructura dental. Por lo anterior, se puede inferir que el tipo de cepillo y el tiempo de cepillado producen una disminución de la microdureza del esmalte. En este estudio observamos cambios en la superficie del esmalte los cuales parecen indicar son debido al tiempo de exposición de las pastas blan-

TABLA III

COMPARACIÓN DE LA RUGOSIDAD Y MICRODUREZA ENTRE LOS TIEMPOS DE EXPOSICIÓN PARA CADA GRUPO DE ESTUDIO.

\begin{tabular}{|c|c|c|c|c|c|c|c|c|c|c|c|c|}
\hline \multirow[b]{2}{*}{ Días } & \multicolumn{2}{|c|}{$\mathrm{Sc}$} & \multicolumn{2}{|c|}{ Co } & \multicolumn{2}{|c|}{$\operatorname{Tr}$} & \multicolumn{2}{|c|}{$\mathrm{Lu}$} & \multicolumn{2}{|c|}{$\mathrm{Cr}$} & \multicolumn{2}{|c|}{$3 \mathrm{D}$} \\
\hline & $\mathrm{R}$ & M & $\mathrm{R}$ & M & $\mathrm{R}$ & M & $\mathrm{R}$ & M & $\mathrm{R}$ & M & $\mathrm{R}$ & M \\
\hline 0 vs 7 & 0,0001 & 1,0000 & 0,0001 & 0,1173 & 0,0001 & 0,0498 & 0,0001 & 0,0354 & 0,0001 & 0,0354 & 0,0001 & 0,0340 \\
\hline vs 14 & 0,0001 & 1,0000 & 0,0001 & 0,0025 & 0,0001 & 0,8494 & 0,0001 & 0,0136 & 0,0001 & 0,0079 & 0,0001 & 0,0040 \\
\hline vs 28 & 0,0001 & 1,0000 & 0,0001 & 0,0001 & 0,0001 & 0,0018 & 0,0001 & & 0,0001 & 0,0368 & 0,0001 & 0,0004 \\
\hline 7 vs 14 & 0,0001 & 1,0000 & 0,3506 & 0,0135 & 0,0001 & 0,6237 & 0,5064 & 0,7637 & 0,1737 & 0,7277 & 0,1329 & 0,4570 \\
\hline 7 vs 28 & 0,0001 & 1,0000 & 0,0104 & 0,0001 & 0,0003 & 0,0397 & 0,0302 & 0,1032 & 0,0791 & 0,6126 & 0,5798 & 0,0068 \\
\hline 14 vs 28 & 0,0001 & 1,0000 & 0,1329 & 0,0001 & 0,2892 & 0,1687 & 0,0001 & 0,0200 & 0,0005 & 0,2820 & 0,1173 & 0,0142 \\
\hline
\end{tabular}

$\mathrm{Se}=$ Sin cepillado, $\mathrm{Co}=$ Control, $\mathrm{Tr}=$ Colgate Triple Acción, $\mathrm{Lu}=$ Colgate Luminous White, $\mathrm{Cr}=\mathrm{Crest}, \mathrm{y}$ 3D $=$ Crest $3 \mathrm{D}, \mathrm{R}=$ Rugosidad, $\mathrm{M}=$ Microdureza.

Vol. 62(1): $63-72,2021$ 
queadoras las cuales afectan la estructura y dureza adamantina y generan diferencias significativas en la microdureza del tejido adamantino aumentándola a los 28 días de exposición y cepillado dental no así en tiempos menores.

Moosavi y col. estudiaron el efecto de los tratamientos posteriores al blanqueamiento y microdureza del esmalte dental in vitro; examinaron, con un microscopio electrónico de barrido, las superficies del esmalte de modelos dentales bovinos mediante diferentes tratamientos e identificaron cambios en la superficie del esmalte el cual mostraba porosidades, depresiones y alteraciones de diversos grados en su superficie, relacionando la dureza del esmalte con la eliminación del contenido mineral del mismo (16). En el presente estudio se utilizaron órganos dentarios humanos sanos extraídos, se analizaron en el microscopio electrónico de barrido y se observó con el uso de pastas blanqueadoras abrasión, patrones de grabado (por el tipo de dentífrico) y desmineralización del tejido adamantino, probablemente por el uso de agentes de remineralización así como agentes de relleno y abrasivos que conducen a estas diferencias. Los resultados muestran al análisis por microscopia de barrido un patrón de desgaste aǵresivo en la superficie adamantina con el cepillado cotidiano sin pasta dental, en el cual se identifican surcos y depresiones por desgaste mecánico producido por las cerdas del cepillo disminuyendo la microdureza probablemente por la pérdida de tejido adamantino, no así con las pastas dentales usadas en el estudio que sugieren un efecto pulidor sobre el esmalte dental. Los hallazgos anteriores indican la importancia del uso de la pasta dental durante el cepillado, mostrando una disminución en la rugosidad de la superficie, evitando el acumulo de película adquirida y preservando la forma y estructura del tejido adamantino.

Karadas y col. en 2015, evaluaron la efectividad de la eliminación de manchas con agentes blanqueadores que contienen diferentes concentraciones de peróxido y el cepi- llado con una pasta dental sin aǵentes blanqueadores durante 8 semanas de tratamiento. Los resultados de su estudio corroboraron los hallazgos reportados por estudios clínicos previos y estudios in vitro, donde la pasta de dientes blanqueadora no indicó diferencias significativas para la eliminación de la mancha extrínseca en la superficie del diente en comparación con la pasta de dientes convencional. Todos los ǵrupos, excepto CWT (pasta de dientes Crest 3D White), fueron efectivos en el blanqueamiento dental en comparación con la pasta dental convencional (17). A diferencia de ese estudio, donde se evaluó solo una crema dental, en este se evaluaron 4 pastas blanqueadoras. Los resultados indican que el dentífrico Crest fue el que causo más abrasión cuando se comparó con los demás grupos de estudio. Las diferencias entre el estudio de Karadas y col. y el nuestro radica en que ellos tiñeron los dientes con té negro, utilizaron cepillos eléctricos y también usaron otros agentes químicos para blanquear como por ejemplo el peróxido de hidrógeno no identificando diferencias significativas al usar la pasta blanqueadora. Comparando con el estudio anterior se encuentran una serie de factores que no concordamos, entre los que se incluyen: las diferencias de protocolo de aplicación de los agentes aclaradores, la tinción de las muestras, la preparación de la muestra, los tiempos de cepillados. En nuestro estudio no se usaron agentes que producen manchas extrínsecas, sin embargo se revisó la abrasión de las pastas sobre el tejido adamantino, se identificaron cambios en el uso de cepillado dental sin pasta vs con pasta, mostrando cambios significativos en la rugosidad de la superficie dental. Al análisis del efecto de los cambios en el uso de las pastas dentales se determinaron cambios significativos a diferentes tiempos unas de otras, lo cual nos indica el potencial de abrasión de cada pasta indicando la posible acción sobre las manchas extrínsecas.

En el estudio de da Silva y col. en 2018, identificaron alteraciones significativas en la rugosidad, estabilidad óptica y translucidez 
del esmalte; observaron un aumento significativo en la rugosidad del tejido adamantino con el cepillado dental y al realizar ciclos con pastas blanqueadoras (8). Los resultados de la presente investigación concuerdan con el autor ya que se identificó aumento en la microdureza dental, cambios topográficos en la superficie adamantina y diferentes patrones de pulido producidos por el uso de las diferentes pastas dentales empleadas en la presente investigación.

Sukran y col. en 2012, concluyeron que los procedimientos de cepillado aumentan la rugosidad de la superficie del esmalte y que el régimen de blanqueamiento realizado con el tratamiento de limpieza, mediante el cepillado con dentífrico blanqueador, disminuyen los valores de dureza. Nuestros resultados concuerdan con los del autor ya que el efecto mecánico del cepillado con o sin pasta dental producen diferentes patrones de rugosidad y dureza en el esmalte dental, ya que la constancia en los ciclos de la acción mecánica del cepillado dental dará como resultado el desǵaste de la superficie observándose diferentes topografías (18).

Finalmente, en esta investigación todos los grupos de estudio presentaron un efecto de pulido. Con base a los resultados obtenidos y de manera inferencial, se recomendaría el uso de la pasta dental Colgate Luminous White para obtener un pulido constante de la superficie adamantina ya que, a los 28 días mostró diferencias significativas en relación con los demás grupos de estudio. Respecto a la microdureza, aunque no se identificaron diferencias significativas, se puede inferir que la pasta dental Crest 3D proporciona un mejor patrón de resistencia en la superficie externa del tejido adamantino. Los resultados obtenidos en este estudio confirman la necesidad no solo de efectuar el cepillado dental sino también la importancia del uso de la pasta dental, la cual proporciona mayor dureza y pulido a la superficie dental. Se sugiere que la elección de la pasta dental sea producto del asesoramiento de un especialista con base a las necesidades específicas de cada individuo.

\section{REFERENCIAS}

1. Tao D, Smith R, Zhang Q, Sun J, Philpotts C, Ricketts S, Naenni M, Joiner A. Tooth whitening evaluation of blue covarine containing toothpastes. J Dent 2017;67:S20-24.

2. Joiner A, Luo W. Tooth colour and whiteness: A review. J Dent 2017;67:S3-10.

3. Kwon S, Wertz P. Review of the mechanism of tooth whitening. J Esthet Restor Dent 2015;27:240-257.

4. Rahardjo A, Gracia E, Riska G, Adiatman M, Maharani D. Potential side effects of whitening toothpaste on enamel roughness and micro hardness. Int J Clin Prev Dent 2016;11(4):239-242.

5. Gutiérrez-llave M, Bernuy-Torres L, Medina-Calderón K, Vadillo-Palacios G. Efecto blanqueador significativo de cinco pastas dentales blanqueadoras. Odontología Sanmarquina 2014;12(1):22-4.

6. Ganss C, Marten J, Hara A, Schlueter N. Toothpastes and enamel erosion/abrasion Impact of active ingredients and the particulate fraction. J Dent 2016;54:62-7.

7. Martínez-Madero E. Eficacia clínica de pastas y colutorios blanqueantes sin peróxidos. Hacia una revisión sistemática. Cient Dent 2016;13(3):199-209.

8. da Silva E, Maia J, Mitraud C, Russo J, Poskus L, Guimaraes J. Can whitening toothpastes maintain the optical stability of enamel over time? J Appl Oral Sci 2018;26(0):1-9.

9. Hara A, Turssi C. Baking soda as an abrasive in toothpastes: Mechanism of action and safety and effectiveness considerations. J Am Dent Assoc 2017;148(11):S27-33.

10. Sabrah A, Lippert F, Kelly A, Hara A. Comparison between radiotracer and surface profile methods for the determination of dentifrice abrasivity. Wear 2013;306:73-79.

11. Salgado-Villa SK, Scougall-Vilchis RJ, Rodríguez-Vilchis LE, Velázquez-Enríquez U. Efecto de un cepillo dental eléctrico con pastas dentales blanqueadoras sobre la superficie del esmalte. Estudio in vitro. Superficies y Vacío 2016; 29(4):116-119.

12. Sabrah A, Turssi C, Lippert F, Eckert G, Kelly A, Hara A. 3D-Image analysis of the impact of toothpaste abrasivity on the progression of simulated non-carious cervical lesions. J Dent 2018;73:1-5.

Vol. 62(1): $63-72,2021$ 
13. Vilhena K, Nogueira B, Fagundes N, Loretto S, Angelica R, Lima R, Silva M, Júnero $\mathrm{S}$. Dental enamel bleached for a prolonged and excessive time: Morphological changes. PLoS One 2019;14(4):1-13.

14. Carey C. Tooth whitening: What we now know. J Evid Based Dent Pr 2014;14:70-76.

15. Maden E, Altun C, Polat G, Basak F. The in vitro evaluation of the effect of Xyliwhite, Probiotic, and the conventional toothpastes on the enamel roughness and microhardness. Niger J Clin Pr 2019;21(3):306-10.

16. Moosavi H, Hakimi N. The effects of fractional CO2 laser, Nano-hydroxyapatite and MI paste on mechanical properties of bovine enamel after bleaching. J Clin Exp Dent 2017;9(12):e1390-1396.
17. Karadas M, Duymus Z. In vitro evaluation of the efficacy of different over-the-counter products on tooth whitening. Braz Dent J 2015;26(4):373-377.

18. Bolay S, Yalcin CF, Gurgan S. Efectos del cepillado de dientes con abrasivos fluorados y dentífricos blanqueadores en la superficie del esmalte humano sin blanquear y blanqueado en términos de rugosidad y dureza: un estudio in vitro. J Contemp Dent Pract 2012;13(5): 584-9. 